\title{
Experimental study and modelling of the water phase change kinetics in soils
}

\author{
A.-L. Lozano, F. Cherblanc, B. Cousin \& J.-C. Bénet \\ Laboratoire de Mécanique et Génie Civil, Université Montpellier 2 - Cc 048, Place Eugène Bataillon, 34095 Montpellier, France
}

\begin{abstract}
Summary
When dealing with porous media, the liquid-gas phase-change is generally considered instantaneous, while a retardation time is observed in the case of hygroscopic soils. So far, little research has been done to characterize the non-equilibrium behaviour of water phase change. Therefore, we propose a macroscopic model of the liquid-gas phase-change rate in porous media, based on the difference of chemical potentials between the liquid and its vapour, which is taken as the driving force. It introduces a phenomenological coefficient that must be determined experimentally. An original experiment able to create a macroscopic non-equilibrium between the liquid and its vapour is described. Analysing the return to equilibrium leads to the determination of the phenomenological phase-change coefficient. Depending on the range of partial vapour pressure, two different behaviours are observed: a linear domain close to equilibrium and a nonlinear one far from equilibrium. The results emphasize the relation between water retention properties in hygroscopic porous media and these phase-change characteristics.
\end{abstract}

\section{Introduction}

At the scale of the liquid-gas interface, the phase-change phenomenon is generally represented by the Knudsen relation (Alty \& McKay, 1935; Knudsen, 1950; Maa, 1967; Fang \& Ward, 1999). This relation, developed from the kinetic theory for ideal gases, defines the maximum theoretical evaporation rate through a unit surface under vacuum conditions (Knudsen, 1950; Knacke \& Stranski, 1956; Eames, 1997). Many experimental investigations (Alty \& McKay, 1935; Maa, 1967) have pointed out that this theoretical relation overpredicts the observed evaporation rate. Therefore, a correcting coefficient, $\varepsilon$, has been introduced. It is defined as the experimental rate of evaporation divided by the theoretical maximum rate, under the same conditions (Knudsen, 1950; Knacke \& Stranski, 1956; Eames, 1997). When a liquid is evaporating into a pressure lower than its own vapour pressure, an additional pressure term is introduced to take into account the actual vapour pressure in the atmosphere surrounding the liquid. This is the so-called Hertz-Knudsen equation:

$$
J_{\text {inter }}=\varepsilon\left(P_{\mathrm{vs}}-P_{\mathrm{v}}\right)\left(\frac{M_{\mathrm{e}}}{2 \pi R T}\right)^{1 / 2}
$$

where $J_{\text {inter }}$ is the interfacial mass flux $\left(\mathrm{kg} \mathrm{m}^{-2} \mathrm{~s}^{-1}\right), P_{\mathrm{v}}$ is the vapour pressure in the gas phase $(\mathrm{Pa}), P_{\mathrm{vs}}$ is the corresponding

Correspondence: F. Cherblanc. E-mail: chb@1mgc.univ-montp2.fr Received 18 April 2007; revised version accepted 17 March 2008 saturating vapour pressure $(\mathrm{Pa})$ and $T$ is the temperature of the liquid surface $(\mathrm{K})$. Various theoretical and experimental research has focused on the evaluation of the evaporation coefficient $\varepsilon$. Empirically derived values for the evaporation coefficient of water have ranged from 0.001 to 1 and it is, therefore, understandable that this coefficient has been the subject of much speculation over the last 60 years, as extensively reviewed by Eames (1997).

When dealing with natural porous media (soil, food products, wood, ...), this relation is affected by various aspects like the local curvature of the gas-liquid interface, which can considerably reduce the phase-change rate (Zhang \& Whang, 2002). Moreover, the hygroscopic nature of such materials manifests itself at a microscopic scale through water adsorption on solid particles. This means that the water cannot be considered to be in a "free" state and the evaporation process requires greater energy exchange.

To be fairly usable in practical applications, a phase-change relation has to be developed at the macroscopic scale. Indeed, the microstructural complexity observed in such materials makes it unrealistic to develop modelling of those phenomena at the pore scale. To perform such upscaling, different mathematical techniques are available, like homogenization (Auriault \& Lewandowska, 1997; Bouddour et al., 1998), volume averaging (Quintard \& Whitaker, 1994) or lattice gas models (Békri et al., 2001). These approaches assume, on the one hand, the existence of a Representative Elementary Volume (REV) sufficiently large to incorporate all the microscopic complexity, 
and on the other hand, complete knowledge of all phenomena occurring at the microscopic scale. When applied to the case of the phase-change phenomenon, the development of an accurate microscopic formulation seems to be a tremendous task, since the influence of adsorptive forces or interfacial curvatures has not been clearly established. Thus, in the upscaling framework, previous theoretical work generally assumes a simple micro-structure (Whitaker, 1977; Moyne \& Perre, 1991), as done with pore-network models (Le Bray \& Prat, 1999; Chen et al., 2005), with a narrow pore-size distribution. In such descriptions, hygroscopic effects cannot be taken into account, since the solid phase is generally considered inert with respect to the liquid phase. At the interface scale, instantaneous phase-change is often assumed by relying on the local equilibrium assumption.

To take into account all the microscopic features, we propose to investigate the gas-liquid phase-change in a soil at the macroscopic scale by means of a phenomenological approach. At this scale, the porous medium is homogenized so that the different phases and constituents are represented by various continua that can interact through exchange terms. Considering the mass balance equations of the liquid and its vapour, they are linked by a mass exchange term, $J\left(\mathrm{~kg} \mathrm{~m}^{-3} \mathrm{~s}^{-1}\right)$, which is associated with the gas-liquid phase-change (Bénet \& Jouanna, 1982; Ruiz \& Bénet, 2001):

$$
\begin{gathered}
\frac{\partial \rho_{1}}{\partial t}=-\left(\rho_{1} v^{k}\right)_{, k}-J, \\
\frac{\partial \rho_{\mathrm{v}}}{\partial t}=-\left(\rho_{\mathrm{v}} v_{\mathrm{v}}^{k}\right)_{, k}+J,
\end{gathered}
$$

where $\rho_{1}$ and $\rho_{\mathrm{v}}\left(\mathrm{kg} \mathrm{m}^{-3}\right)$ are, respectively, the apparent density of water and its vapour, and $v_{1}^{k}$ and $v^{k}{ }_{\mathrm{v}}\left(\mathrm{m} \mathrm{s}^{-1}\right)$ are, respectively, the intrinsic velocity of water and its vapour. The phase-change rate $J$ is thus a volumetric scalar flux, representing the mass of water transforming from a liquid state to a vapour state by unit volume and unit time.

One of the major interests of such development is to improve the transport modelling of pollutants, like Volatile Organic Compounds (VOCs), migrating into the vadose zone of soils. Regarding the aqueous-gaseous mass transfer modelling of NAPL, a huge literature is available. Many experimental analyses have led to the development of first-order correlations as functions of soil and flow characteristics (Armstrong et al., 1994; Szatkowski et al., 1995; Wilkins et al., 1995; Chao et al., 1998). These correlations have been introduced in various multiphase transport models to simulate pollution remediation techniques based on air injection or extraction (Mulligan \& Yong, 2004; Maier \& Grathwohl, 2005; Serrano et al., 2006; Rahbeh \& Mohtar, 2007). However, in these investigations, the rate-limited behaviour results from microscopic convective processes in the gas-phase, i.e., the mass transfer depends on the gas flow rate. Thus, none of these models can account for a rate-limited phase-change when both the liquid- and gas- phase are immobile, since they degenerate in this situation. On the other hand, the natural liquid-gas phase-change without any external driving forces (gas pressure gradient, forced convection, ...) has been generally considered instantaneous when compared to the relaxation time of the other transport phenomena (convection, diffusion, ...). This is fairly the case in capillary porous media where hygroscopic effects are negligible. Nevertheless, under certain conditions in an isolated non-saturated soil, a volatilization retardation time has been observed (Bénet \& Jouanna, 1982; Armstrong et al., 1994). Previous experimental investigations have dealt with the phasechange of water and heptane in a clayey silty sand (Bénet \& Jouanna, 1982; Ruiz \& Bénet, 2001; Chammari et al., 2003) and they have underlined the influence of the liquid content and temperature. Obviously, high temperature enhances the phasechange and heptane is much more volatile than water. Most of all, it has been clearly brought out that the phase-change rate in a hygroscopic soil is not infinite.

However, these previous researches focused on situations close to the equilibrium where classical linear Thermodynamics of Irreversible Processes (TIP) apply, i.e., phenomena can be linearized. The objective of this study is twofold, first, to investigate gas-liquid phase-change far from the equilibrium conditions, i.e., beyond the linear domain, second, to analyse the influence of the hygroscopic characteristics of soil on the phase-change phenomenon.

For the readability of this paper, the basic ideas used to develop the phenomenological relation of phase-change are summarized in the next section. Then, the experimental device and materials are fully described. The numerical data processing is detailed in the following section, where the experimental results are also presented. Finally, these results are discussed with respect to the linear and the non-linear domains.

\section{Macroscopic relation of phase-change}

A natural soil can be idealized by a triphasic medium constituted of an undeformable solid skeleton made of grains, a liquid phase which is pure water in this study, and the gas phase containing a mixture of dry air and water vapour. Assuming a rigid structure is a strong hypothesis since evaporation and dehydration processes in non-consolidated soils generally lead to global shrinkage. This also affects the water thermodynamic state, as shown by Braudeau \& Mohtar (2004). However, we focus our attention on the hygroscopic domain where these effects are negligible. Moreover, the quantity of water evaporated during the experiments presented in the next section is small enough to fairly consider a constant water content, meaning that the phenomena can be decoupled.

Using a phenomenological approach, this heterogeneous system is homogenized by considering three continua that interact. At this macroscopic scale, it is assumed that there is a thermodynamic non-equilibrium between the water and its 
vapour, represented by a chemical potential jump between phases. Developing the mass, momentum, energy and entropy balances in a triphasic porous medium leads to the expression of the internal production rate of entropy due to phase-change, $\chi(\mathrm{J}$ $\mathrm{K}^{-1} \mathrm{~m}^{-3} \mathrm{~s}^{-1}$ ), as (Knacke \& Stranski, 1956; Bénet \& Jouanna, 1982; De Groot \& Mazur, 1984; Taylor \& Krishna, 1993; Kuiken, 1994; Bedeaux \& Kjelstrup, 2004, 2005; Ruiz \& Bénet, 2001):

$$
\chi=J \frac{\mu_{1}-\mu_{\mathrm{v}}}{T} \geq 0
$$

where $J$ is the volumetric phase-change rate and $\mu_{1}$ and $\mu_{\mathrm{v}}(\mathrm{J}$ $\mathrm{kg}^{-1}$ ) are, respectively, the chemical potentials of water in the liquid state and in the vapour state. The difference of the chemical potentials between the water and its vapour thus becomes the driving force of the phase-change phenomenon. At the macroscopic scale, this phenomenon is considered isothermal which justifies the use of a single temperature field. This assumption will be experimentally verified later in this paper.

Near equilibrium, in the framework of linear TIP, the thermodynamic flux is expressed as a linear function of the thermodynamic force (Bénet \& Jouanna, 1982; Ruiz \& Bénet, 2001):

$$
J=L(w, T) \frac{\mu_{1}-\mu_{\mathrm{v}}}{T} .
$$

where the water content, $w$, is defined as the ratio of the apparent densities of water and solid phase. This relation introduces a phenomenological coefficient, $L\left(\mathrm{~kg} \mathrm{~K} \mathrm{~s} \mathrm{~m}^{-5}\right)$, that depends on the state variables, such as the water content, $w$, and the temperature, $T$, but also on the soil characteristics, such as the porosity, the texture, the nature of materials, ... This coefficient has to be determined experimentally, and will be explained in the next section.

To be useful in practice, the chemical potential must be expressed as a function of an experimentally measurable quantity, like the vapour pressure. In the gas phase, the chemical potential of the vapour is given by:

$$
\mu_{\mathrm{v}}=\mu_{\mathrm{v}}^{0}(T)+\frac{R T}{M_{1}} \ln \left(\frac{P_{\mathrm{v}}}{P^{o}}\right),
$$

where $R$ is the ideal gas constant $\left(\mathrm{J} \mathrm{mol}^{-1} \mathrm{~K}^{-1}\right), M_{1}$ is the liquid phase molar mass $\left(\mathrm{kg} \mathrm{mol}^{-1}\right), P_{\mathrm{v}}$ is the partial pressure of vapour and $P^{o}$ is a reference pressure. In hygroscopic porous media at a small water content, the liquid phase is partially adsorbed to the grains' surface. Thus, the chemical potential of water depends on the surface forces and cannot be derived rigorously (Bolt \& Miller, 1958; Nitao \& Bear, 1996). However, it can be expressed through the chemical potential of its vapour in equilibrium conditions by writing:

$$
\mu_{1}=\mu_{\mathrm{veq}}=\mu_{\mathrm{v}}^{0}(T)+\frac{R T}{M_{1}} \ln \left(\frac{P_{\mathrm{veq}}}{P^{0}}\right) .
$$

The equilibrium partial pressure of vapour, $P_{\text {veq }}$, depends on the activity of the liquid phase, $a(w, T)$, and on the saturating vapour pressure, $P_{\mathrm{vs}}(T)$, by:

$$
P_{\mathrm{veq}}=a(w, T) P_{\mathrm{vs}}(T) .
$$

The activity is deduced explicitly from experimental desorption isotherms (Figure 1).

Using these expressions of chemical potentials (Equations 6 and 7), the phenomenological relation of phase-change, in isothermal conditions, is written (Bénet \& Jouanna, 1982; Ruiz \& Bénet, 2001):

$$
J=L(w, T) \frac{R}{M_{1}} \ln \left(\frac{P_{\mathrm{v}}}{P_{\text {veq }}}\right) .
$$

In the former relation, the ratio of the vapour pressure divided by its equilibrium value plays the role of a non-equilibrium criterion. Since this relation is developed in the framework of the linear TIP, it is valid near equilibrium, when $P_{\mathrm{v}}$ is close to its equilibrium value $P_{\text {veq }}$, with a constant phenomenological coefficient $L$, i.e., it does not depend on the generalized thermodynamic force represented by the ratio of vapour pressure divided by its equilibrium value. Previous experimental and numerical investigations dealing with the natural drying of soil columns pointed out that phase-change occurs generally in conditions that cannot be considered to be close to equilibrium (Chammari et al., 2003, 2008). Indeed, the partial vapour pressure could easily reach less than half of its equilibrium value, where the linearity of Equation (9) is no longer valid. To take into account this non-linear behaviour, a dependence of the phenomenological coefficient, $L$, on the partial vapour pressure, $P_{\mathrm{v}}$, is introduced:

$$
J=L\left(w, T, \frac{P_{\mathrm{v}}}{P_{\mathrm{veq}}}\right) \frac{R}{M_{1}} \ln \left(\frac{P_{\mathrm{v}}}{P_{\mathrm{veq}}}\right) .
$$

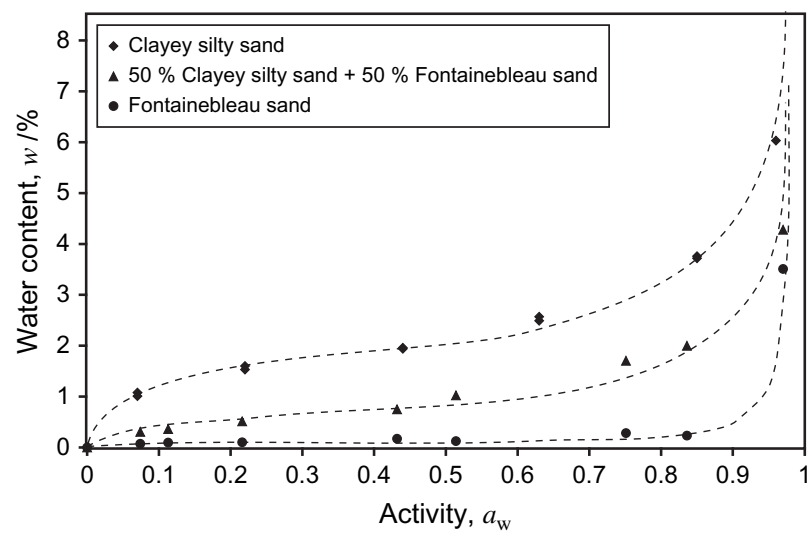

Figure 1 Desorption isotherm at $T=30^{\circ} \mathrm{C}$ of the two kinds of soil under investigation. The desorption isotherm obtained with pure sand is also given for comparison. 
In the more general case, the phase-change rate depends non-linearly on the vapour partial pressure. Through Equation (10), this non-linear dependence is divided into two parts: the phenomenological coefficient, $L$, and the logarithm function. This splitting is arbitrary. Nevertheless, it has already experimentally been shown that, in the neighbourhood of equilibrium, the coefficient $L$ is constant and does not depend on the vapour pressure. Consequently, to ensure the continuity between both behaviours, the non-linear formulation of phase-change is similar to the one developed in the linear TIP framework. At this point, the main objective of this paper is to investigate experimentally the validity of this model, and its relevance to hygroscopic soils. This will be done by determining the phase-change coefficient $L$ over a large range of water contents and vapour pressures, in order to identify clearly the two domains: close to and far from equilibrium. This should justify the relation (10) as an extension of the formulation (9).

\section{Experimental study of phase-change}

This section deals with the experimental determination of the phenomenological coefficient $L$ introduced in Equations (9) and (10). The phase-change phenomenon must be activated in a soil sample, large enough to be considered as a REV. To isolate this phenomenon from other transport phenomena (diffusion, convection, ...), several experimental constraints are required:

- the uniformity of the soil state variables so as to neglect any gradient (porosity, temperature, water content, ...),

- a constant temperature to obtain the isothermal conditions assumed in the theoretical development.

Starting from an equilibrium state, the experimental principle consists of extracting a fraction of the gas phase from the soil sample. By doing this, the partial vapour pressure in the pores drops below its equilibrium value, $P_{\text {veq }}$, stimulating the bulk evaporation phenomenon. The return back to thermodynamic equilibrium manifests itself by a total gas pressure increase. Analysing this return to equilibrium allows us to determine the phase-change rate represented by the phenomenological coefficient, $L$.

\section{Materials}

With the purpose of showing the influence of the micro-structure on phase-change behaviour, two kinds of soils are used:

- A natural soil, clayey silty sand (CSS), from the bank of the Hérault river (south of France). This soil is classified as SC-CL according to the USCS. The liquid and plastic limits are respectively $25 \%$ and $14.5 \%$. Sand, silt and clay fraction are $72 \%, 18 \%$ and $10 \%$ respectively. The mineralogical analysis based on X-ray diffraction techniques gives $50 \%$ of calcite, $40 \%$ of quartz and $10 \%$ of clay (Saix et al., 2000). The organic matter content has been found to be negligible. The clayey frac- tion, although existing in a small proportion, plays a predominant role with respect to the water retention characteristics. The physical properties are summarized in Table 1.

- A mixture made of 50\% CSS and 50\% sand. The sand used is a fine siliceous sand from Fontainebleau (France) with a uniform granulometry $(315 \mu \mathrm{m}<d<500 \mu \mathrm{m})$.

The thermodynamic state of water in soil is characterized by its activity $a(w, T)$, which relates the saturating vapour pressure to the equilibrium vapour pressure (Equation 8). The water activity can be deduced directly from a desorption isotherm, which gives the equilibrium water content of a soil sample as a function of the surrounding relative humidity. For the two kinds of soil presented above, the water activities are plotted in Figure 1. The activity obtained with pure sand is also given for comparison. A clear difference between these curves is observed, emphasizing the major role played by the clayey fraction on water retention.

\section{Experimental device and procedure}

The complete experimental device is presented in Figure 2. A cylindrical soil sample (2) (30-mm diameter and 12-mm high) is used. The soil is first dried at $105^{\circ} \mathrm{C}$ for 24 hours. The required amounts of soil (18 g) and demineralized water are added using a high-precision balance $\left(10^{-4} \mathrm{~g}\right)$. After mixing, it is stored in a waterproof container for 24 hours to ensure the homogeneity of the water content. Then, the wet soil is compacted in a cylindrical ring by means of a hydraulic press in order to reach a dry density of $1500 \mathrm{~kg} \mathrm{~m}^{-3}$, which corresponds to a porosity of $43 \%$. The water content $(w)$ is calculated by differential weighing:

- after the mixing of the dry soil and the water,

- at the end of the experiment.

These two measurements ensure that the amount of water evaporated is negligible, justifying the assumption of a constant water content during the experiment.

The sensor (1) used to record the temperature and the pressure at scheduled time steps is autonomous and programmable (type NanoVACQ from TMI Orion, Montpellier, France). A vacuum pump linked to a pressure controller holds the absolute pressure at $20 \mathrm{kPa}$ in a vacuum vessel (4). A valve (3) allows us, on one hand, to put the soil sample in contact with the vacuum vessel, and, on the other hand, to completely isolate the sample. The whole device is placed in a thermoregulated bath in order to maintain a constant temperature.

The experimental process includes three steps:

- Phase 1 - Thermal equilibrium: The device and sample are put in the thermo-regulated bath for one hour until thermal equilibrium is obtained.

- Phase 2 - Extraction of the gas phase: For 3 seconds, the soil sample is connected to the vacuum vessel through the valve. This stage aims to lower the partial vapour pressure below its equilibrium value, which creates a thermodynamic nonequilibrium between the liquid phase and its vapour. With 
Figure 2 Experimental device for the determination of the phase-change coefficient, $L$.

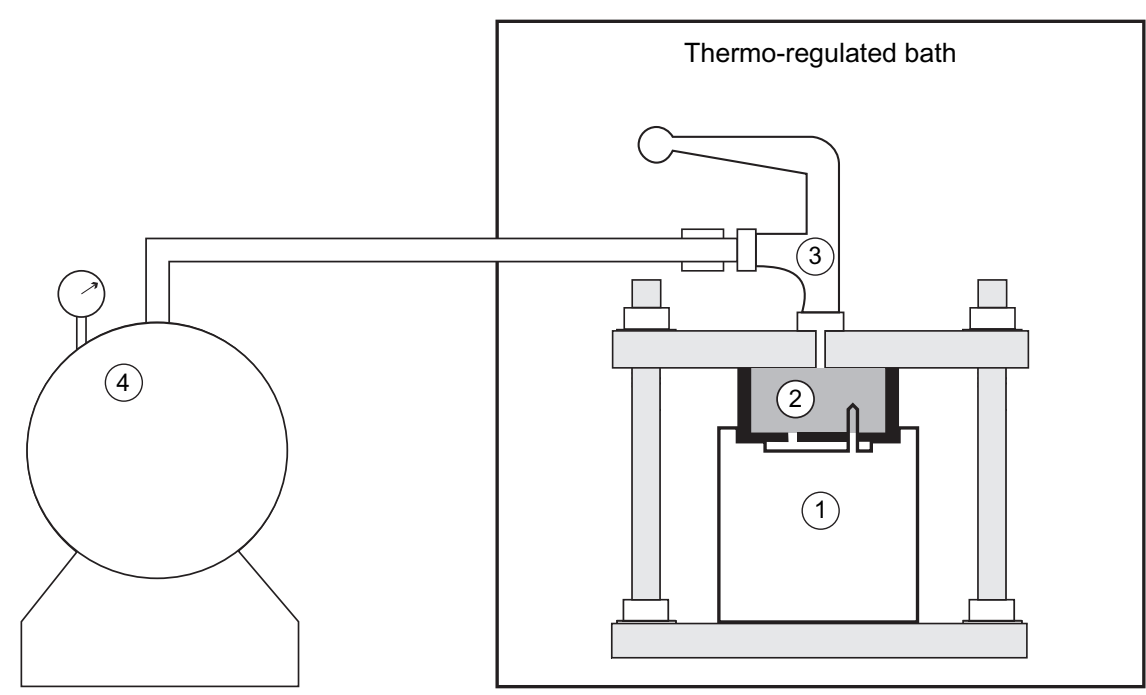

(1) Pressure and temperature sensor

(3) 3-way valve

(2) Soil sample
(4) Vacuum vessel respect to the time required for the whole experiment, this phase is considered instantaneous.

- Phase 3 - Return to equilibrium: After closing the valve, the soil sample is completely insulated and then evolves as a thermodynamically closed system in isothermal conditions. The temperature, $T$, and total gas pressure, $P_{\mathrm{g}}$, are recorded during this phase.

\section{Experimental observations}

In the case of a CSS sample at $T=30^{\circ} \mathrm{C}$ and $w=2.10 \%$, the evolution of temperature, $T$, and total gas pressure, $P_{\mathrm{g}}$, are plotted versus time in Figure (3).

The total gas pressure variation is approximately $\Delta P_{\mathrm{g}}=1500$ $P a$ (Figure 3b). This is less than the equilibrium partial vapour pressure, which is $P_{\text {veq }}=2130 P a$ at $w=2.10 \%$ (Equation 8 ). This means that the first second of the evaporation process cannot be caught since the phase-change flux rate is too high at the beginning.

Figure 3 a shows that the change in temperature is less than $0.05 K$. This temperature variation can be evaluated from energy exchange considerations. Knowing the initial $\left(P_{\mathrm{v}}=\right.$ $\left.0.2 P_{\text {veq }}\right)$ and final $\left(P_{\mathrm{v}}=P_{\text {veq }}\right)$ partial vapour pressures allows

Table 1 Characteristic values of the soil solid phase

\begin{tabular}{llrr}
\hline Physical properties & Symbol & Value & Unit \\
\hline Real mass density & $\rho^{*}$ & 2650 & $\mathrm{~kg} \mathrm{~m}^{-3}$ \\
Apparent mass density & $\rho_{\mathrm{s}}$ & 1500 & $\mathrm{~kg} \mathrm{~m}^{-3}$ \\
Average porosity & $n$ & 0.43 & $\mathrm{~m}^{3} \mathrm{~m}^{-3}$ \\
$\quad$ in a dry state & & & \\
\hline
\end{tabular}

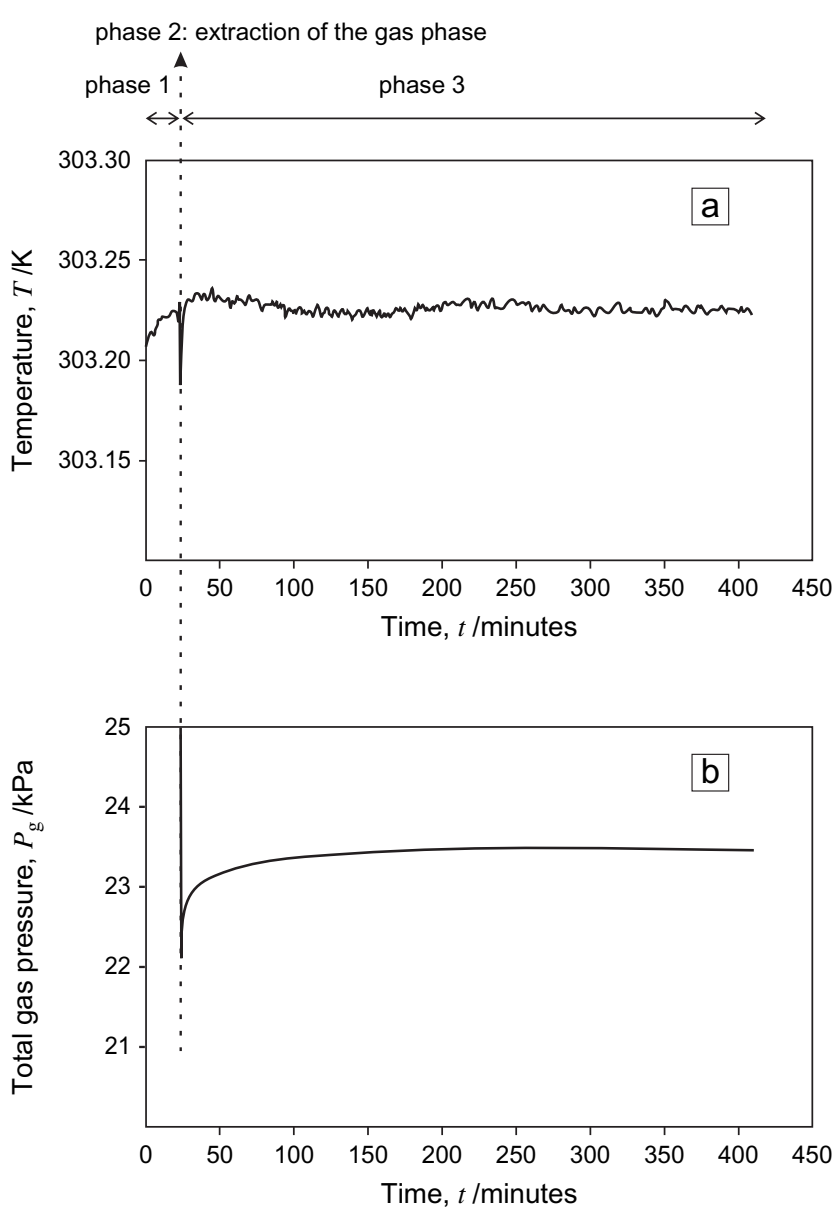

Figure 3 Temperature and pressure evolution with a CSS sample at $T=30^{\circ} \mathrm{C}$ and $w=2.10 \%$. a) Temperature, $T,-$ b) Total gas pressure, $P_{\mathrm{g}}$. 
us to determine the amount of water that changes phase. Regarding the experimental results presented above, we have (in $\mathrm{kg} \mathrm{m}^{-3}$ ):

$$
\Delta \rho_{\mathrm{v}}=\frac{\eta_{\mathrm{g}} M_{1}}{R T} P_{\mathrm{veq}}(1-0.2)=5.23 \times 10^{-3},
$$

where $\eta_{\mathrm{g}}$ is is the volume fraction of the gas phase. The evaporation of this quantity of water can be represented by a twostep process. First, the water adsorbed on solid surfaces changes into a "free" state. In this transformation, energy is required to break up the adsorptive interactions between the liquid and solid phases. Secondly, this amount of liquid changes into a vapour state. This transformation involves heat transfer. Generally, the energy required in the first step is negligible with respect to the latent heat of evaporation; some details are given in the final section. Therefore, regarding the experiment presented above, the required energy is given (in $\mathrm{J} \mathrm{m}^{-3}$ ) by:

$$
E=L_{\mathrm{v}} \Delta \rho_{\mathrm{v}}=11.8 \times 10^{3}
$$

where $L_{\mathrm{v}}$ is the evaporation latent heat $\left(\mathrm{J} \mathrm{kg}^{-1}\right)$. This amount of energy is taken from the soil and causes a drop in temperature:

$$
Q=\left(\rho_{\mathrm{s}} C_{\mathrm{s}}+\rho_{1} C_{1}\right) \Delta T,
$$

where $C_{\mathrm{s}}$ and $C_{1}\left(\mathrm{~J} \mathrm{~K}^{-1} \mathrm{~kg}^{-1}\right)$ are respectively the heat capacities of the solid and liquid phase. We have assumed that the calorific energy stored in the gas phase is negligible. Relating Equation (12) to Equation (13) leads to an estimate of a decrease in temperature of less than $0.03 \mathrm{~K}$. This is comparable to the experimental observations, justifying the isothermal conditions assumed in the theoretical development in the former section.

\section{Experimental results}

The experimental procedure adopted aims to activate an homogeneous phase-change in the sample. Under these conditions, the convective fluxes in the mass balances Equations (2) and (3) are cancelled. Using the ideal gas model, the vapour mass balance equation is written in terms of partial pressure:

$$
\frac{\partial P_{\mathrm{v}}}{\partial t}=-L \frac{R^{2} T}{\eta_{\mathrm{g}} M_{1}^{2}} \ln \left(\frac{P_{\mathrm{v}}}{P_{\text {veq }}}\right) .
$$

Our objective at this point is to validate the relation of the phase-change (Equation 10) and to determine the value of the phenomenological coefficient, $L$, based on measurements of $P_{\mathrm{g}}$ and $T$ (Figure 3).

The partial vapour pressure, $P_{\mathrm{v}}$, can be deduced from the experimental total gas pressure, $P_{\mathrm{g}}$. After closing the valve, the soil sample is a closed system at a constant temperature. Therefore, the amount of dry air in the pores remains constant and its partial pressure, $P_{\mathrm{a}}$, is considered invariable. This pressure $P_{\mathrm{a}}$ can be evaluated when equilibrium is reached at the end of the experiment. In these conditions, $P_{\mathrm{v}}=P_{\text {veq }}$, and by assuming that the ideal gas model is valid for a mixture, we have:

$$
t \rightarrow \infty ; P_{\mathrm{a}}=\left(P_{\mathrm{g}}\right)_{t \rightarrow \infty}-P_{\mathrm{veq}}
$$

During one experiment, the temperature and water content are considered constant, and the equilibrium vapour pressure, $P_{\text {veq }}$, is given by Equation (8). Thus, the partial vapour pressure can be calculated by:

$$
P_{\mathrm{v}}=P_{\mathrm{g}}-P_{\mathrm{a}}
$$

Regarding the results presented in Figure 3, the corresponding evolution of vapour pressure is plotted in Figure 4. To compute the pressure derivative appearing in Equation (14), this experimental curve is smoothed using a Savitsky-Golay algorithm (Press et al., 1992). At this stage, the phenomenological coefficient, $L$, can be determined at each time step by Equation (14). In this relation, the ratio of the vapour pressure divided by its equilibrium value, i.e. $P_{\mathrm{v}} / P_{\text {veq }}$, corresponds to the generalized thermodynamic force that governs the phasechange phenomenon (Equation 5). It can also be seen as a non-equilibrium criterion indicating the experimental conditions, far from or close to equilibrium. From that perspective, the phenomenological phase-change coefficient, $L$, can be plotted as a function of this non-equilibrium criterion (Figure 5). This allows us to distinguish two domains. Close to equilibrium, where the ratio of vapour pressure is $>0.67$, the phasechange behaves as a linear phenomenon with a constant mass exchange coefficient. Actually, this limit defines the domain of validity where linear TIP applies. The linearity close to equilibrium has already been observed using a different data processing method (Bénet \& Jouanna, 1982; Ruiz \& Bénet, 2001; Chammari et al., 2003). Below this limit, as non-equilibrium increases, non-linear behaviour is observed, while the phasechange kinetics are strongly enhanced.

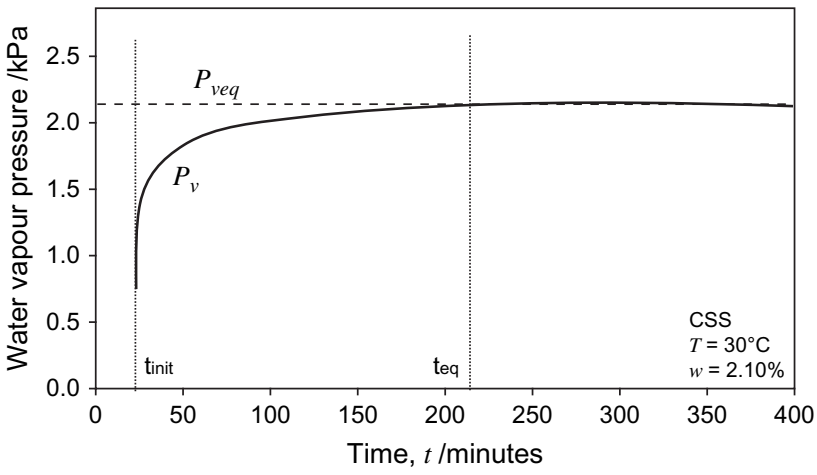

Figure 4 Evolution of $P_{\mathrm{v}}$ and $P_{\mathrm{veq}}$ for an experiment with a CSS sample at $T=30^{\circ} \mathrm{C}$ and $w=2.10 \%$. 


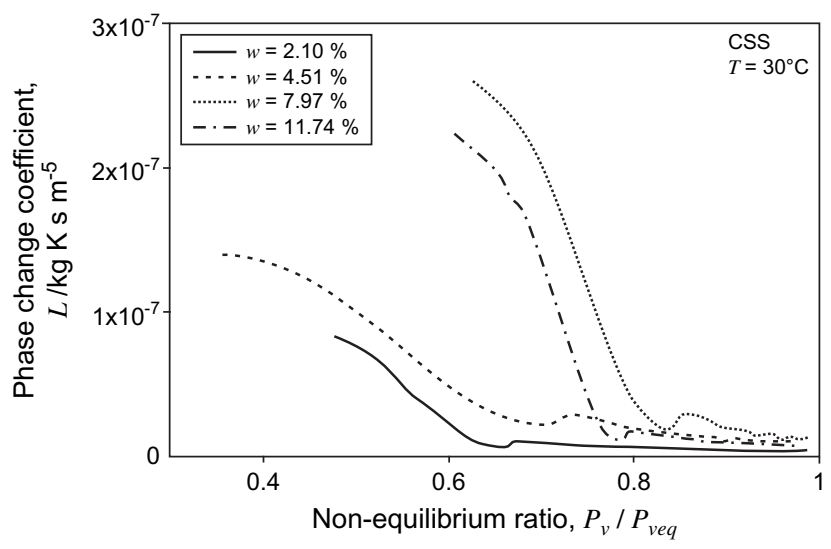

Figure 5 Evolutions of the phenomenological coefficient versus $P_{\mathrm{v}} / P_{\text {veq }}$ with a CSS sample at $T=30^{\circ} \mathrm{C}$ and various water contens, $w$.

For the range of water contents investigated, similar twodomain behaviours have always been observed (Figure 5). Consequently, as a first approximation, a simple model is proposed (Figure 6):

- a linear domain: $L=L_{\mathrm{eq}}$ if $r \leq \frac{P_{\mathrm{v}}}{P_{\mathrm{veq}}} \leq 1$,

- a non-linear domain: $L=L_{\mathrm{eq}}+k\left(r-\frac{P_{\mathrm{v}}}{P_{\text {veq }}}\right)$ if $0 \leq \frac{P_{\mathrm{v}}}{P_{\text {veq }}} \leq r$.

Thus, three parameters represent the medium reactivity to a thermodynamic non-equilibrium:

- $L_{\text {eq }}$, the phase-change coefficient close to equilibrium,

- $r$, the transition ratio between the domain far from equilibrium and the domain close to equilibrium,

- $k$, the slope in the domain far from equilibrium.

This model is definitely too simplistic to represent accurately the complexity of the phase-change phenomenon far from equilibrium. Nevertheless, as a first approximation, it can give a global indication on how the phase-change kinetics depends on the non-equilibrium $P_{\mathrm{v}} / P_{\text {veq. }}$. From our point of view, the objective

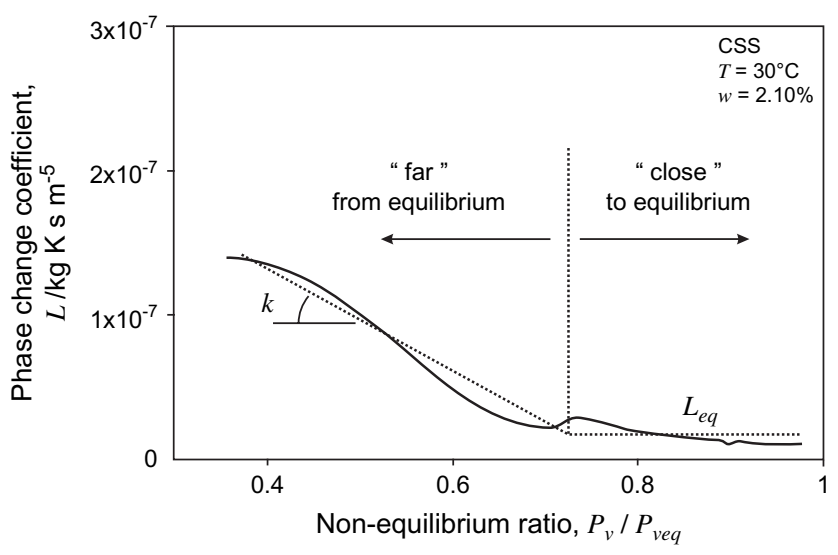

Figure 6 Evolution of the phenomenological coefficient versus $P_{\mathrm{v}} / P_{\text {veq }}$ (solid line) and the two-domain model (dashed line). is above all to discuss the influence of the water content and texture, rather than propose a precise descriptive model. Thus, the question about the quality of this model is beyond the scope of this paper.

This procedure has been applied for each case, to explore the whole range of water contents, varying from $2 \%$ to $12 \%$, for the two kinds of soil under investigation. At $<2 \%$ water content, the soil is too powdery to yield a usable sample. Above $12 \%$, the gas phase is occluded and the phase-change cannot be activated.

\section{Discussion}

For both materials under investigation: the CSS and the mixture of $50 \% \mathrm{CSS}+50 \%$ sand, the entire set of experimental results is presented in Figures 7, 8 and 9. On these Figures, the dashed lines are free-hand drawn, and are given only to indicate the global trend. These curves will be discussed by referring to the two domains defined in the former section.

\section{Linear Domain}

Close to equilibrium, when $r \leq P_{\mathrm{v}} / P_{\text {veq }} \leq 1$, the phenomenological coefficient, $L_{\text {eq }}$, is given in Figure (7) as a function of the water content. Like the results presented by Bénet \& Jouanna (1982) and Ruiz \& Bénet (2001), some bell-shaped curves are observed. The water content corresponding to the maximum is noted, $w_{\text {opt }}$. This typical shape can be analysed by referring to the hydrological state of the soil:

- $0 \leq w \leq w_{\text {opt }}$ : this range of water content corresponds to the hygroscopic domain (Figure 1) where the water activity deviates from 1 . In this state, the water is partially adsorbed on the solid phase. Thus, the less the water content, the stronger the adsorption interactions. This leads to a decrease in the phasechange kinetics as $w$ tends towards 0 .

- $w_{\text {opt }} \leq w$ : the water is globally in a "free" state, and hygroscopic effects are negligible. As the water content increases,

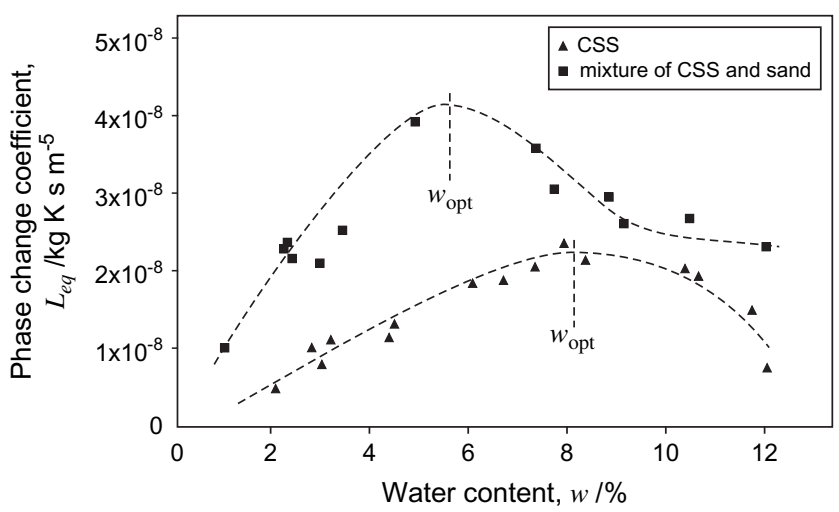

Figure 7 Phenomenological coefficient close to equilibrium, $L_{\mathrm{eq}}$, versus the water content, $w$, for the two kinds of soil. The dashed lines are given for indication. 


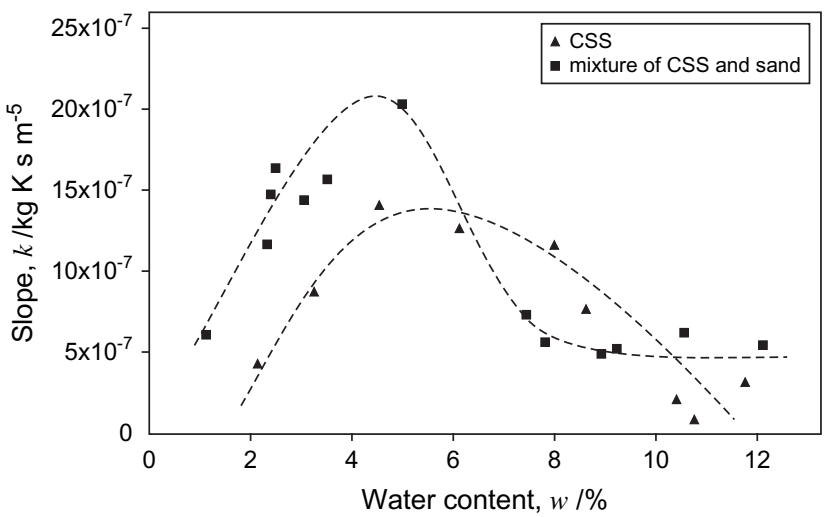

Figure 8 Parameter $k$ as a function of the water content, $w$, for the two kinds of soil. The dashed lines are given for indication.

the gas-volume fraction decreases, leading to a smaller liquidgas interfacial surface available for evaporation. At the same time, the vapour diffusion mechanism in the gas phase is partially blocked off. For a water content around 14\%, the gas phase is occluded and the phase-change cannot be activated.

As presented above, the hygroscopicity and the phase-change characteristics are intimately related. One can note that these two characteristics are not of the same kind. The first qualifies an equilibrium situation, while the second describes the kinetics resulting from non-equilibrium conditions. Nevertheless, it is not surprising to observe that these characteristics are correlated since they involve the same microscopic physical quantity: the adsorption energy. This water-solid binding energy can be evaluated through the chemical potential of the water phase. The chemical potential difference between an adsorbed state and a "free" state is given by:

$$
\begin{aligned}
\Delta \mu & =\mu_{\text {free }}-\mu_{\text {adsorbed }} \\
& =\mu_{1}^{0}+\frac{R T}{M_{1}} \ln P_{\mathrm{vs}}-\left(\mu_{1}^{0}+\frac{R T}{M_{1}} \ln P_{\mathrm{veq}}\right) \\
& =-\frac{R T}{M_{1}} \ln \frac{P_{\mathrm{veq}}}{P_{\mathrm{vs}}} \\
& =-\frac{R T}{M_{1}} \ln [a(w, T)] .
\end{aligned}
$$

This binding energy is directly related to the activity of water, $a(w, T)$, given by Equation (8) and Figure (1). For a water content of $2 \%$ and $4 \%$ respectively, the water activities in the CSS are 0.45 and 0.88 , which leads to a binding energy of $E=112$ $\mathrm{kJkg}^{-1}$ and $E=17.9 \mathrm{kJkg}^{-1}$. Thereby, when the water content goes from $2 \%$ to $4 \%$, the binding energy is 6 times smaller. This is in agreement with the faster phase-change kinetics observed in Figure (7) since the phenomenological coefficient,
$L$, is doubled. The more hygroscopic the material, the smaller its phase-change coefficient.

Comparison of the results obtained on different materials confirms this assertion. A fraction of sand has been mixed with the CSS in order to decrease its hygroscopicity, as presented in Figure (1). The phase-change coefficient, $L_{\mathrm{eq}}$, measured with this second material is also plotted in Figure (7). For a water content of $4 \%$, the activity of water in this soil is 0.98 , corresponding to a binding energy of $E=2.83 \mathrm{kJkg}^{-1}$. Thus, by reducing the clayey fraction, the binding energy is divided by 6 and the phase-change coefficient is multiplied by 2 , leading to the same ratio as identified in the former paragraph. Figure 1 shows that adding some sand leads to a reduction of the hygroscopic domain. This is confirmed in Figure 7 where the maximum $w_{\text {opt }}$ is shifted to the left in the case of the mixture.

Those results emphasize the predominant role played by the clayey fraction in the retention and phase-change properties. Even with a relatively small clayey fraction, $10 \%$ in this study, about $90 \%$ of the water is stored in the clayey structure. At the microscopic scale, the kinetic-molecular theory can account for these experimental observations. The evaporation of "free" water molecules can be spontaneous but is mainly stimulated by the shock of other molecules evolving in the gas phase (Matsumoto, 1998). Adsorption interactions applied to water molecules would affect their availability to participate in molecular shocks and evaporation.

\section{Non-Linear Domain}

The limit of validity of the linear domain is defined by $r$, in terms of a ratio of the vapour pressure to its equilibrium value. Outside the linear domain, i.e., far from equilibrium, the phasechange kinetics are characterized by the slope $k$. These two characteristics are presented as functions of the water content in Figures (8) and (9) for both materials. The parameter $k$ behaves similarly to the phase-change coefficient $L_{\text {eq }}$, manifesting some bell-shaped variations. The same conclusions as given in the previous section can be formulated. The microstructure of the soil and its hygroscopic features play a dominating role in the phase-change phenomenon. By adding some sand, identical effects are observed on both characteristics, i.e., a global increase and a shift of the maximum to the left.

On the other hand, the transition criterion, $r$, does not seem to be affected by the type of soil or by the water content (Figure 9).

The cause of this non-linear behaviour has not yet been clearly established. However, the explanation should be sought at a smaller scale. A natural soil is an extremely heterogeneous medium at multiple scales. The clayey fraction creates some very fine porous aggregates $(\approx 0.1 \mu \mathrm{m})$ embedded in a larger heterogeneous structure made of sand grains $(\approx 100 \mu \mathrm{m})$ (Bartoli et al., 2005). With clayey silt at a small water content, a double-porosity structure is often encountered (Delage et al., 1996). At equilibrium, the liquid water is adsorbed in this 


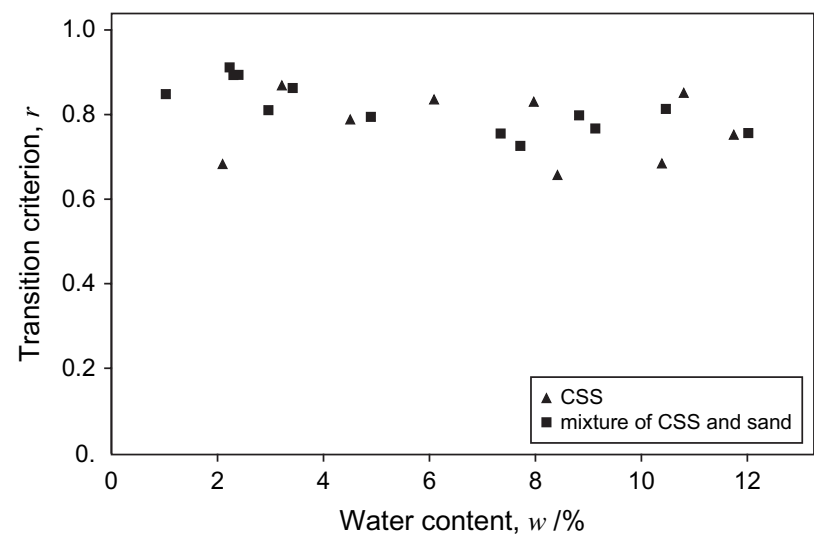

Figure 9 Transition criterion, $r$, as a function of the water content, $w$, for the two kinds of soil.

complex system by some mechanisms that depend on the nature of the solid phase materials and their surface (clay, quartz, calcite) (Mitchell, 1993). Thus, even if the second principle of thermodynamics imposes the uniformity of the water chemical potential in all its forms, its distribution should be very complex. Any disturbance of this equilibrium by mass exchange with the environment will distort the uniformity of the water chemical potential. This generally comes with microscopic temperature gradients resulting from interface cooling. At the pore scale, the return to equilibrium consists of the movement of liquid water, or vapour, from places with a high chemical potential to places with a low chemical potential. Local thermal transfers should also occur. These mechanisms are not instantaneous and the combination of them may result in the retardation times and complex non-linear behaviour observed at the macroscopic scale.

\section{Implications}

Usual descriptions of liquid-gas mass transfer proposed in the literature are formulated in terms of first-order kinetics based on concentration differences (Armstrong et al., 1994; Szatkowski et al., 1995; Wilkins et al., 1995; Chao et al., 1998). This means that the phase-change rate increases linearly as the vapour content decreases. Close to equilibrium conditions, the logarithm that appears in Equation (10) can be linearized, thus allowing us to rewrite this model in term of first-order kinetics. In this situation, the phase-change model (Equation 10) matches the usual mass transfer descriptions with a constant mass transfer coefficient, $L$, that depends on the experimental conditions (materials, temperature, ...). Since we focused on natural attenuation without gas-forced convection, the experimental coefficient characterized in this work can be interpreted as the limit at which the gas velocity tends toward zero. However, the experimental results presented here clearly demonstrate that, outside of a limited linear domain, the phase-change phenomenon exhibits considerable non-linear behaviour that cannot be caught by a simple first-order representation.

These results could have a large implication if put in the context of water resources. In the dry season, where small water contents are generally observed, the liquid water retained in soils is entrapped by adsorptive or capillary forces. Indeed, the soilwater system is in a hygroscopic or pendular state. This means that the liquid phase is no longer continuous and the concepts of pressure field or capillary potential are meaningless. In these situations, the chemical potential becomes the key parameter to describe water transport. Taking into account the thermodynamic non-equilibrium between the liquid and gas phase leads to the development of a water and moisture transport model where the liquid-gas phase-change plays a central role (Chammari et al., 2003, 2008). In the particular case of a hygroscopic soil at small water content, the limiting phenomenon that governs the water transport processes could be the phase-change rather than vapour diffusion. This point of view can help in interpreting the drying kinetics or infiltration profiles observed experimentally. Indeed, classical evapo-transpiration models propose some simple correlations of the quantity of water evaporated in the first soil layer as a function of environmental parameters (temperature, relative humidity, wind speed, ...). They are empirically developed and the influence of the soil texture or the boundary humidity cannot be established reliably. Revisiting these models by relying on a microscale formulation of water transport processes should provide some refinements leading to more accurate evapo-transpiration models.

\section{Conclusions}

This paper focuses on the liquid-gas phase-change kinetics in porous media when non-equilibrium conditions are encountered. Using the chemical potential difference of water between the liquid and the vapour state as the driving force leads to the development of a phase-change relation that introduces a phenomenological coefficient $L$. This modelling is characterized by two domains: a domain described as linear in which the phasechange coefficient is constant, and a domain described as nonlinear in which the phase-change coefficient depends linearly on the partial vapour pressure.

The experimental measures carried out on two hygroscopic soils allow the phase-change characteristics to be determined for the whole range of water content. Their dependencies on the water content shows the influence of the soil texture, and demonstrate the competition between two microscopic features: first, the liquid-solid binding energy resulting from adsorption phenomena, and, secondly, the liquid-gas interfacial surface available for evaporation. The first feature has a decreasing influence when the water content increases, whereas the second one becomes predominant for higher water content. Indeed, the water activity in soil and the phase-change characteristics 
appear to be intimately related, even though the first property is determined in equilibrium conditions while the second one characterizes a non-equilibrium evolution.

From an upscaling point of view, the phenomenological coefficient $L$ encapsulates all the underlying microscopic phenomena such as: interfacial phase-change, vapour diffusion, thermal conduction, filtration inside clay aggregates, ... The characteristic times associated with heat transfer or vapour diffusion are too low to explain the retardation time observed in our experiments. Nevertheless, the microscopic phasechange law (Equation 1) could be drastically affected by adsorption interactions, electrical double-layer or local interfacial curvatures. Further microscopic thermodynamic developments should bring some essential physical descriptions of phase-change. Anyway, the experiments presented here do not make it possible to reach a conclusion on the microscopic dominating phenomena that govern the phase-change kinetics. However, this study shows that the partial vapour pressure, the temperature and the water content are relevant macroscopic variables to model the phase-change in hygroscopic porous media.

\section{References}

Alty, T. \& MacKay, C.A. 1935. The accommodation coefficient and the evaporation coefficient of water. Proceedings of the Royal Society of London, A149, 104-116.

Armstrong, J., Frind, E. \& McClellan, R. 1994. Non-equilibrium mass transfer between the vapor, aqueous, and solid phases in unsaturated soils during vapor extraction. Water Resources Research, 30, 355-368.

Auriault, J. \& Lewandowska, J. 1997. Modelling of pollutant migration in porous media with interfacial transfer: Local equilibrium/ non-equilibrium. Mechanics of Cohesive Frictional Materials, 2, 205221.

Bartoli, F., Genevois-Gomendy, V., Royer, J.J., Niquet, S., Vivier, H. \& Grayson, R. 2005. A multiscale study of silty soil structure. European Journal of Soil Science, 56, 207-224.

Bedeaux, D. \& Kjelstrup, S. 2004. Irreversible thermodynamics a tool to describe phase transitions far from global equilibrium. Chemical Engineering Science, 59, 109-118.

Bedeaux, D. \& Kjelstrup, S. 2005. Heat, mass and charge transport and chemical reactions at surfaces. International Journal of Thermodynamics, 8, 25-41.

Bénet, J.-C. \& Jouanna, P. 1982. Phenomenological relation of phasechange of water in a porous medium: Experimental verification and measurement of the phenomenological coefficient. International Journal of Heat and Mass Transfer, 25, 1747-1754.

Békri, S., Vizika, O., Thovert, J.F. \& Adler, P.M. 2001. Binary twophase flow with phase-change in porous media. International Journal of Multiphase Flow, 27, 477-526.

Bolt, G. \& Miller, R. 1958. Calculation of total and component potentials of water in soil. Transactions of the American Geophysical Union, 39, 917-928.

Bouddour, A., Auriault, J., Mhamdi-Alaoui, M. \& Bloch, J.F. 1998. Heat and mass transfer in wet porous media in presence of eva- poration-condensation. International Journal of Heat and Mass Transfer, 41, 2263-2277.

Braudeau, E. \& Mohtar, R.H. 2004. Water potential in nonrigid unsaturated soil-water medium. Water Resources Research, 40, doi:10.1029/2004WR003119.

Chammari, A., Naon, B., Cherblanc, F. \& Bénet, J.-C. 2003. Transfert d'eau en sol aride avec changement de phase: Water transport with phase-change at low water content. Comptes Rendus de l'Académie des Sciences, 331, 759-765.

Chammari, A., Naon, B., Cherblanc, F., Cousin, B. \& Bénet, J.-C. 2008. Interpreting the drying kinetics of a soil using a macroscopic thermodynamic non-equilibrium of water between the liquid and vapour phase. Drying Technology, 26, in press.

Chao, K., Ong, S.K. \& Protopapas, A. 1998. Water-to-air mass transfer of VOCs: laboratory-scale air sparging system. Journal of Environmental Engineering, 124, 1054-1060.

Chen, M., Rossen, W. \& Yortsos, Y. 2005. The flow and displacement in porous media of fluids with yield stress. Chemical Engineering Science, 60, 4183-4202.

De Groot, S.R. \& Mazur, P. 1984. Non Equilibrium Thermodynamics. North-Holland Pub. Co.1962, re-edited by Dover Publications Inc, New York.

Delage, P., Audiguier, M., Cui, Y.J. \& Howat, M. 1996. Microstructure of a compacted silt. Canadian Geotechnical Journal, 33, 150-158.

Eames, I. W., Marr, N. J. \& Sabir, H. 1997. The evaporation coefficient of water: A review. International Journal of Heat and Mass Transfer, 40, 2963-2973.

Fang, G. \& Ward, C. A. 1999. Temperature measured close to the interface of an evaporating liquid. Physical Review, 59, 417-428.

Knacke, O. \& Stranski, I. N. 1956. The mechanism of evaporation. Progress in Metal Physics, 6, 181-235.

Knudsen, M. 1950. The Kinetic Theory of Gases. Methuen, London.

Kuiken 1994. Thermodynamics of Irreversible Processes. Wiley, Chichester.

Le Bray, Y. \& Prat, M. 1999. Three-dimensional pore network simulation of drying in capillary porous media. International Journal of Heat and Mass Transfer, 42, 4207-4224.

Maa, J. 1967. Evaporation coefficient of liquids. Industrial \& Engineering Chemistry Fundamentals, 6, 504-518.

Maier, U. \& Grathwohl, P. 2005. Natural attenuation of volatile hydrocarbons in the unsaturated zone - modelling for the Vaerlose field site. IAHS-AISH Publication, 297, 296-304.

Matsumoto, M. 1998. Molecular dynamics of fluid phase-change. Fluid Phase Equilibria, 144, 307-314.

Mitchell, J. K. 1993. Fundamentals of Soil Behaviour. Wiley, New York.

Moyne, C. \& Perre, P. 1991. Processes related to drying. Part I. Theoretical model. Drying Technology, 9, 1135-1152.

Mulligan, C. \& Yong, R. 2004. Natural attenuation of contaminated soils. Environment International, 30, 587-601.

Nitao, J. \& Bear, J. 1996. Potentials and their role in transport in porous media. Water Resources Research, 32, 225-250.

Press, W., Teukolsky, S. \& Vetterling, W. 1992. Numerical Recipes in C. The Art of Scientific Computing, Cambridge University Press, Cambridge.

Quintard, M. \& Whitaker, S. 1994. Convection, dispersion, and interfacial transport of contaminants: Homogeneous porous media. Advances in Water Resources, 17, 221-239.

Rahbeh, M. E. \& Mohtar, M. H. 2007. Application of multiphase transport models to field remediation by air sparging and soil vapor extraction. Journal of Hazardous Materials, 143, 156-170. 
Ruiz, T. \& Bénet, J. -C. 2001. phase-change in a heterogeneous medium: Comparison between the vaporisation of water and heptane in an unsaturated soil at two temperatures. Transport in Porous Media, 44, 337-353.

Saix, C., Devillers, Ph. \& El Youssoufi, M. S. 2000. Eléments de couplage thermomécanique dans la consolidation de sols non saturés. Canadian Geotechnical Journal, 37, 308-317.

Serrano, A., Gallego, M. \& González, R. 2006. Assessment of natural attenuation of volatile aromatic hydrocarbons in agricultural soil contaminated with diesel fuel. Environmental Pollution, 144, 203-209.

Szatkowski, A., Imhoff, P. T. \& Miller, C. T. 1995. Development of a correlation for aqueous-vapor phase mass transfer in porous media. Journal of Contaminant Hydrology, 18, 85-106.
Taylor, R. \& Krishna, R. 1993. Multicomponent Mass Transfer. Wiley, New York.

Wilkins, M. D., Abriola, L. M. \& Pennell, K. D. 1995. An experimental investigation of rate-limited nonaqueous phase liquid volatilization in unsaturated porous media: steady state mass transfer. Water Resources Research, 31, 2159-2172.

Whitaker, S. 1977. Simultaneous Heat, Mass and Momentum Transfer in Porous Media: A Theory of Drying. Advances in Heat Transfer, 13, 119-203.

Zhang, J. \& Wang, B. 2002. Effect of capillarity at liquid-vapor interface on phase-change without surfactant. International Journal of Heat and Mass Transfer, 45, 2689-2694. 